\title{
Result Target Coding System
}

National Cancer Institute

\section{Source}

National Cancer Institute. Result Target Coding System. NCI Thesaurus. Code C93660.

The coding system to use for recording results for the associated activity or evaluation. 